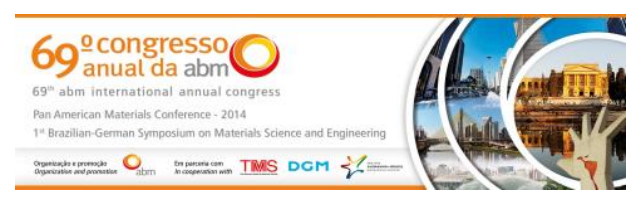

Tema: Lavra e tratamento de minérios

\title{
ESTUDO PRELIMINAR DA DETERMINAÇÃO DO TEOR DE CINZAS DE CARVÃO A PARTIR DA REFLECTÂNCIA ATRAVÉS DA TEORIA DE KUBELKA-MUNK*
}

\author{
Leandro Gustavo Mendes de Jesus ${ }^{1}$ \\ Ítalo Gomes Gonçalves ${ }^{2}$ \\ Carlos Otávio Petter ${ }^{3}$
}

\section{Resumo}

A teoria de Kubelka-Munk é bastante genérica e abrangente. A sua aplicabilidade já foi estudada nas mais diversas áreas, como tintas especiais [1], plásticos [2,3], produtos farmacêuticos [4] e alimentícios [5], e também no caolim [6-8]. A partir da teoria pode se relacionar a reflectância difusa com a função de Kubelka-Munk É também notável que carvões com elevados teores de cinza possuem propriedades colorimétricas diferentes de carvões com baixos teores de cinza. Em função dessa notável diferença acredita-se poder quantificar a quantidade de cinzas a partir da reflectância em diversos comprimentos de onda. Para isso foi elaborado um roteiro de retirada da amostra e confecção de pastilhas de carvão pulverizado onde foram medidos a reflectância e o percentual de cinzas. A partir dessas medidas foi possível a obtenção de equações que modelam a função de Kubelka-Munk em função do percentual de cinzas. Apesar do baixo número de amostras coletadas foi possível se obter funções de Kubelka-Munk que se encaixaram nos dados coletados. Por fim, foi possível concluir que há possibilidade de inferir o teor de cinzas de carvão por meio da reflectância.

Palavras-chave: Determinação do percentual de cinzas; Reflectância difusa do carvão; Teoria de Kubelka-Munk.

\section{PRELIMINARY STUDY OF DETERMINATION OF ASH CONTENT OF COAL FROM REFLECTANCE USING THE THEORY KUBELKA-MUNK}

\section{Abstract}

Kubelka - Munk theory is very general and comprehensive theory. Its applicability has been studied in several areas, such as special inks [1], plastics [2,3], pharmaceuticals products [4] and food industry [5], and also in the kaolin [6-8]. From the theory can relate the diffuse reflectance with the Kubelka-Munk function is also notable that coals with high ash content have different colorimetric properties of coals with low ash content. Due to this remarkable difference is believed to be able to quantify the ash content from the diffuse reflectance at various wavelengths. For this coal was sampled and preparation of pulverized coal tablets which were measured reflectance and ash content was produced. From these measurements it was possible to obtain the equations that model the Kubelka-Munk function based on ash content. Despite the low number of samples collected was possible to obtain functions Kubelka-Munk which fited the data collected. Finally, as a conclusion, it is possible to infer the ash content of coal by reflectance.

Keywords: Ash content determination; Diffuse reflectance of coal, Kubelka-Munk theory.

1 Engenheiro metalúrgico, Professor, COMET, Instituto Federal de Educação, Ciência e Tecnologia de Mato Grosso do Sul, Corumbá, MS, Brasil.

2 Engenheiro de Minas, Mestre em Engenharia de Minas, Materiais e Metalúrgica, Professor, DEMIM, Universidade Federal do Pampa, Caçapava do Sul, RS, Brasil.

3 Engenheiro metalúrgico, Doutor em Engenharia de Minas, Professor, DEMIN, Universidade Federal do Rio Grande do Sul, Porto Alegre, RS, Brasil.

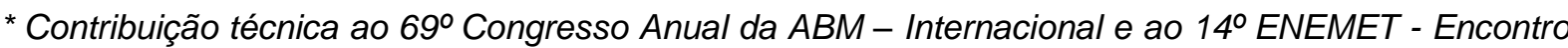
Nacional de Estudantes de Engenharia Metalúrgica, de Materiais e de Minas, 21 a 25 de julho de 2014, São Paulo, SP, Brasil.
} 


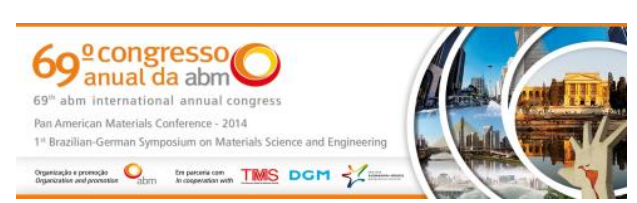

\section{INTRODUÇÃO}

O carvão é maciçamente empregado na geração de energia e na produção de aço, é utilizado como combustível em usinas termelétricas e na indústria siderúrgica como agente redutor. Segundo Abreu [9] na natureza, o carvão se apresenta como uma rocha sedimentar combustível, formada a partir de determinados detritos vegetais, podendo ser encontrado em diferentes estados de conservação e tendo sofrido soterramento e compactação em bacias originalmente pouco profundas.

Também é notável o fato de que minérios com diversos constituintes, todos eles com propriedades diferenciadas e com teores diferenciados de cada um dos constituintes resultam propriedades distintas entre si. O carvão, uma vez que possui diversos constituintes e todos eles com diferentes propriedades, o que torna evidente que teores de cinzas diferentes conduzem a diferença de teores entre os constituinte e por consequência propriedades diferentes. Durante o trabalho o carvão será convencionado como possuindo dois constituintes: cinzas e matéria orgânica. Com relação a propriedades como a reflectância difusa de carvões com alto teor de cinzas são notavelmente diferente de carvões com baixo teor de cinzas. Isto se reflete claramente mesmo ao olhar empírico de que o carvão mineral de baixo teor de cinzas apresenta coloração escura, já os carvões com alto teor de cinzas possuem coloração mais clara. Esta conclusão visual que qualquer observador pode obter é ratificada durante o trabalho.

A teoria de Kubelka-Munk (K-M), por sua vez, é utilizada em diversos segmentos industriais. A principal consequência da teoria é que esta culmina em uma função, denominada função de K-M, que transforma uma grandeza não aditiva, a reflectância difusa, em uma grandeza aditiva. Com isso, a partir da reflectância dos diversos constituintes de uma mistura pura e por intermédio da função de K-M é possível inferir teores de cada um dos constituintes em função da função de K-M.

No caso do estudo em questão deseja-se avaliar a viabilidade de obtenção do percentual de cinzas a partir da reflectância, ou seja, fazer o caminho inverso, através de uma medida do espectro de reflectância determinar a concentração dos diversos minerais contaminantes do carvão como já foi realizado para o caolin por Gonçalves em 2009 [10].

\section{MATERIAIS E MÉTODOS}

O carvão utilizado para quantificar as cinzas a partir da teoria de K-M foi o carvão da Copelmi Mineração Ldta. Foram retiradas amostras de três camadas estratigráficas distintas: as camadas I1 e M camada onde o material era predominantemente de matéria orgânica e uma camada onde o material era predominantemente siltitos, vide figura 1. As amostras foram retiradas diretamente da frente de lavra fazendo-se utilização de uma picareta.

\footnotetext{
* Contribuição técnica ao $69^{\circ}$ Congresso Anual da ABM - Internacional e ao 14ํㅡㄹ ENEMET - Encontro Nacional de Estudantes de Engenharia Metalúrgica, de Materiais e de Minas, 21 a 25 de julho de 2014, São Paulo, SP, Brasil.
} 


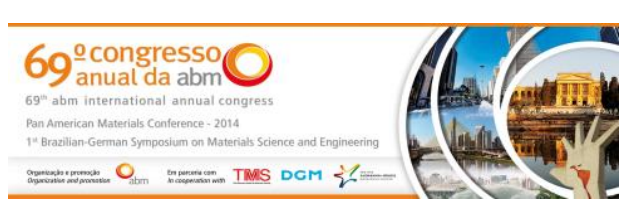

$\mathrm{Na}$ etapa de afunda-flutua o material foi submetido ao ensaio em 4 densidades diferentes, conforme norma NBR 8738, para gerar sub-alíquotas de 5 densidades diferentes (figura 3). Quanto maior a densidade maior o percentual de minerais de ganga (mais densos), logo, maior o percentual de cinzas.

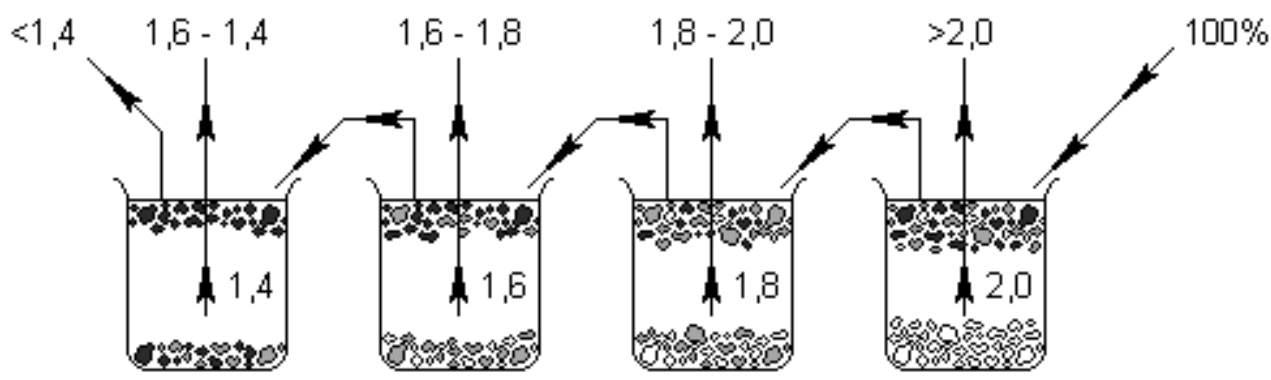

Figura 3: esquema dos ensaios de afunda-flutua, unidades em $\mathrm{kg} / \mathrm{dm}^{3}$.

Posteriormente as amostras foram moídas para ficar na granulometria adequada para o ensaio de determinação de cinzas bem como confecção de pastilhas para medidas de reflectância. Com as 5 amostras de cada incremento foram feitos ensaios para determinação de cinzas de carvão mineral em base seca, segundo norma NBR 8289 e NBR 8293 e, paralelamente, foram realizadas medidas de reflectância isto possibilitou a criação de um modelo a partir das equações de K-M.

Apesar da teoria empregada ser bastante utilizada, geralmente é difícil fazê-lo da maneira como foi concebida, devido a dificuldades práticas na determinação das constantes necessárias. Isto costuma ser resolvido utilizando-se simplificações da teoria original, que envolvem a suposição de que algumas variáveis sejam constantes dentro de certas condições, ou é atribuído um valor arbitrário para algumas constantes. Estas simplificações costumam funcionar quando os contaminantes ocorrem em concentrações bastante baixas e os materiais que formam a base da mistura são constantes. Infelizmente estas duas suposições não são válidas para o carvão, que apresenta contaminantes em altas concentrações e a sua granulometria (logo, suas propriedades óticas) varia. Sendo assim torna-se necessário um procedimento que possibilite a utilização da teoria de K-M sem simplificações, já proposto por Gonçalves em 2009, o que demanda tanto a utilização de novas técnicas experimentais quanto de fórmulas matemáticas para o cálculo de todas as constantes.

Para isto, após o cálculo do percentual de cinzas, para tentar realizar alguma correlação entre reflectância e percentual de cinzas é necessário calcular o $F\left(R_{\infty}\right)$, também conhecida como função de K-M. Isso se torna necessário devido ao fato de que, como já citado, a reflectância não é uma grandeza aditiva ao contrário da função de K-M, por isso, utiliza-se da função abaixo (equação 1) para criação de um modelo:

$$
\frac{K}{S}=\frac{\left(1-R_{\infty}\right)^{2}}{2 R_{\infty}} \equiv F\left(R_{\infty}\right)
$$

onde $\mathrm{K}$ é o coeficiente de absorção de luz por unidade de comprimento e $\mathrm{S}$ coeficiente de espalhamento e $\mathrm{R}_{\infty}$ representa a reflectância de uma camada espessa de minério compactado.

A partir da obtenção de $F\left(R_{\infty}\right)$ e da fração mássica de cinzas foi possível traçar um gráfico onde o eixo das abcissas é representado pela fração mássica de cinzas e o eixo das ordenadas é representado pela função de $\mathrm{K}-\mathrm{M}, F\left(R_{\infty}\right)$ exposta na equação 2. Posteriormente utilizou-se da equação abaixo (método de linha de

* Contribuição técnica ao $69^{\circ}$ Congresso Anual da ABM - Internacional e ao 14ํㅡㄹ ENEMET - Encontro Nacional de Estudantes de Engenharia Metalúrgica, de Materiais e de Minas, 21 a 25 de julho de 2014, São Paulo, SP, Brasil. 


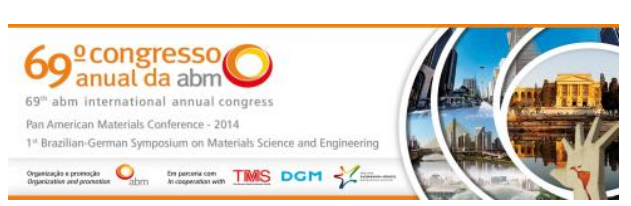

conexão, proposto por Gonçalves [10]) para criação do modelo com a minimização de quadrados dos erros utilizando-se de ferramentas computacionais.

$$
F\left(R_{\infty}\right)=\frac{F_{C}+\left(\alpha_{C z} F_{C Z}-F_{C}\right) c_{C Z}}{1+\left(\propto_{C Z}-1\right) c_{C Z}}
$$

A variável $\propto_{C z C}$ corresponde a taxa é de espalhamento das cinzas em relação a material orgânico. A taxa de espalhamento é facilmente medida e depende somente de $\boldsymbol{F}_{\boldsymbol{C}}$ e $\boldsymbol{F}_{\boldsymbol{c}}$ (obtido a partir da reflectância de cada material em seu estado puro).

\section{RESULTADOS E DISCUSSÃO}

O resultados das medidas realizadas para cada uma das sub-alíquotas está exposto na figura 4. A leitura da reflectância via espectrofotômetro gerou 31 medidas de reflectância para cada comprimento de onde distinto a cada $10 \mathrm{~nm}$ de $400 \mathrm{~nm}$ até 700nm.

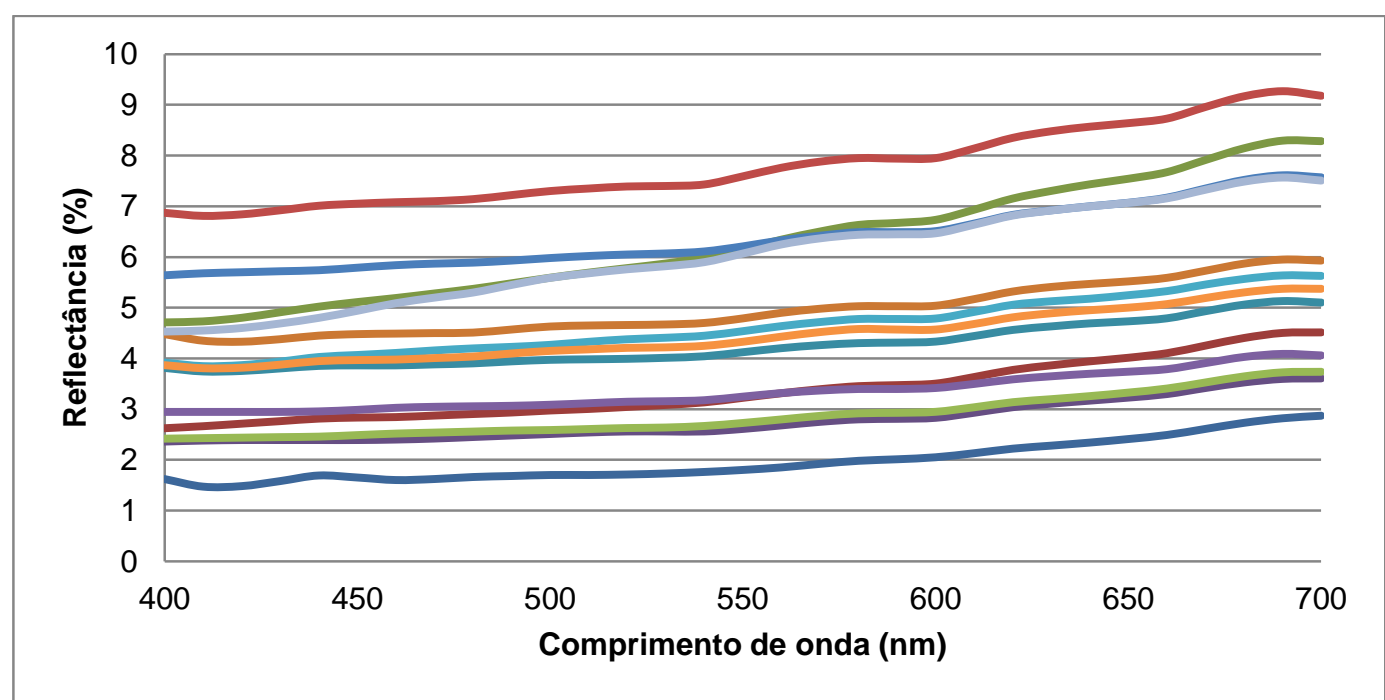

Figura 4: Espectro de reflectância gerado a partir das medidas da reflectância em 31 comprimentos de onda diferentes.

A partir da criação do modelo, por intermédio das etapas já expostas foi possível se obter as constantes para cada um dos comprimentos de onda (figura 5). Sendo assim, a partir das medidas de reflectância para cada um dos comprimentos de onda, foi possível se obter as constantes para cada comprimento de onda e, como resultado, um modelo para referente ao respectivo comprimento de onda. A escolha da exposição dos modelos em $700 \mathrm{~nm}$ se deu em função do fato de que a constante $F_{c}$ convergia para um mesmo valor nas leituras em $700 \mathrm{~nm}$ e gerava um modelo mais consistente e possibilitou, ao fim do trabalho, que fosse feito um modelo agrupando os dados de todas as camadas.

\footnotetext{
* Contribuição técnica ao $69^{\circ}$ Congresso Anual da ABM - Internacional e ao 14ํㅡㄹ ENEMET - Encontro Nacional de Estudantes de Engenharia Metalúrgica, de Materiais e de Minas, 21 a 25 de julho de 2014, São Paulo, SP, Brasil.
} 

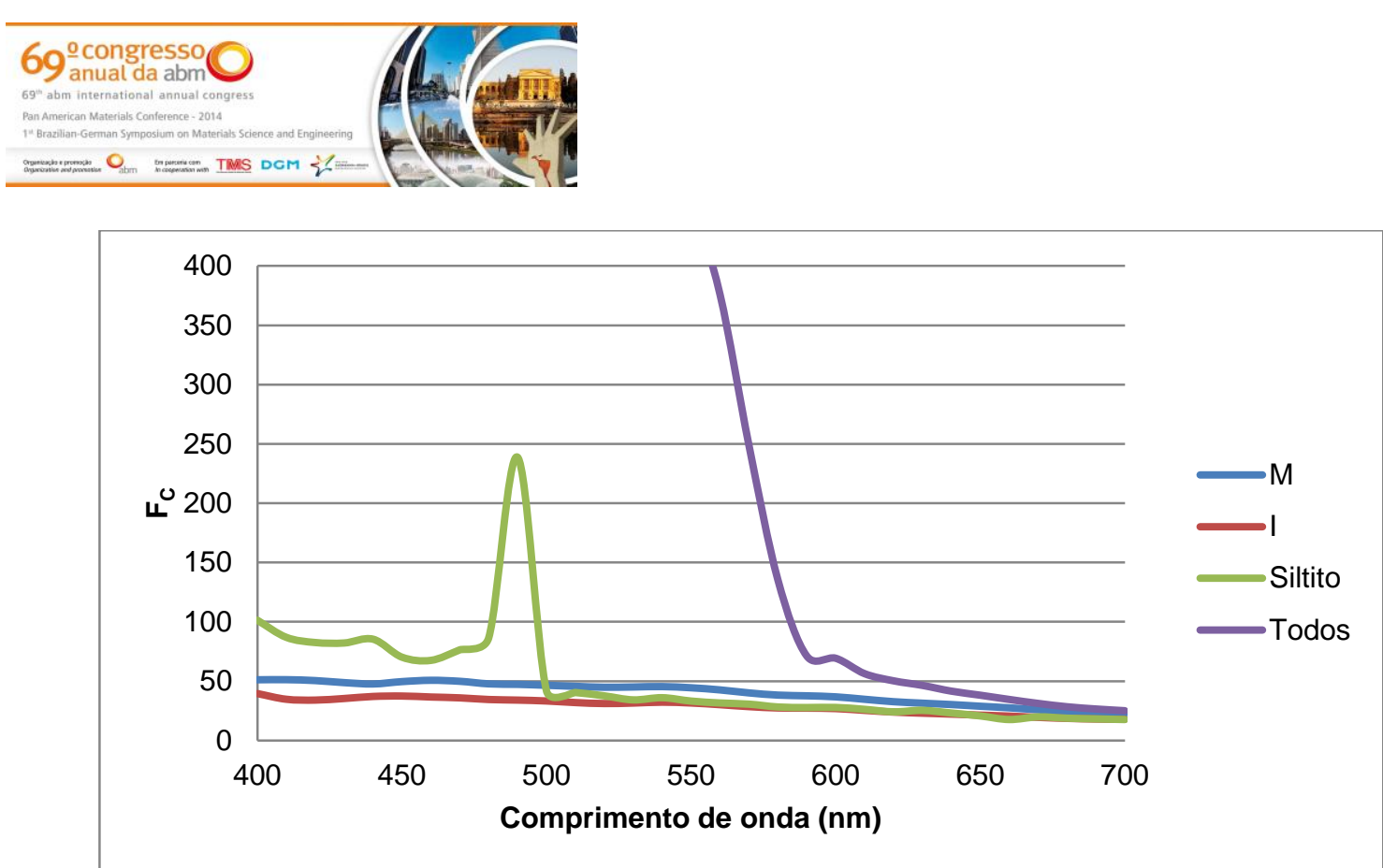

Figura 5: Gráfico da constante $F_{c}$ em função dos diversos comprimentos de onda medidos.

O primeiro modelo gerado foi o modelo da camada $M$ que contou com poucos pontos (3 pontos) devido a baixa quantidade em massa gerada nos ensaios de afunda-flutua de duas faixas de densidades. Como resultado o modelo para as amostras da camada M está exposto na figura 6 .

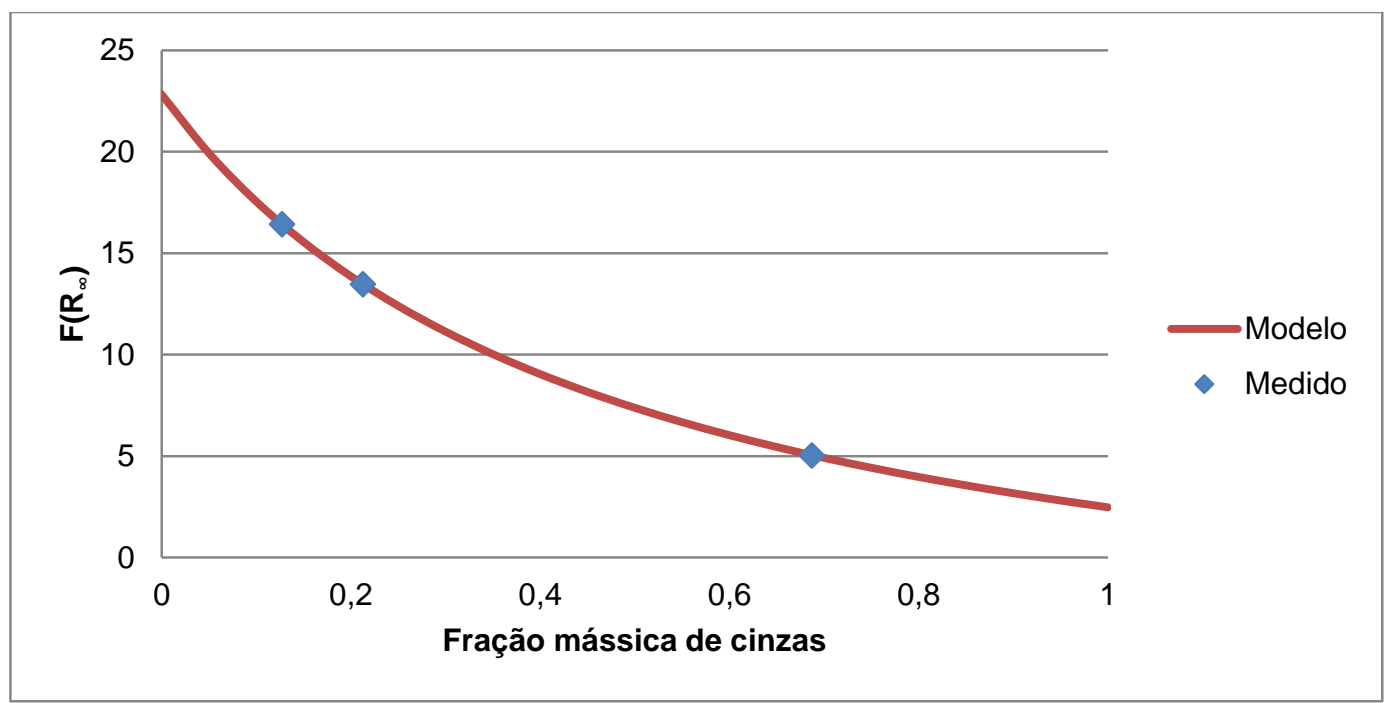

Figura 6: Modelo gerado a partir das amostras da camada $M$ com $F\left(R_{\infty}\right)$ em $700 \mathrm{~nm}$.

O segundo modelo gerado foi o modelo da camada 11 que contou com 5 pontos. Como resultado o modelo para as amostras da camada l1 está exposto na figura 7.

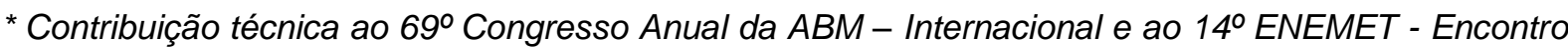
Nacional de Estudantes de Engenharia Metalúrgica, de Materiais e de Minas, 21 a 25 de julho de 2014, São Paulo, SP, Brasil.
} 

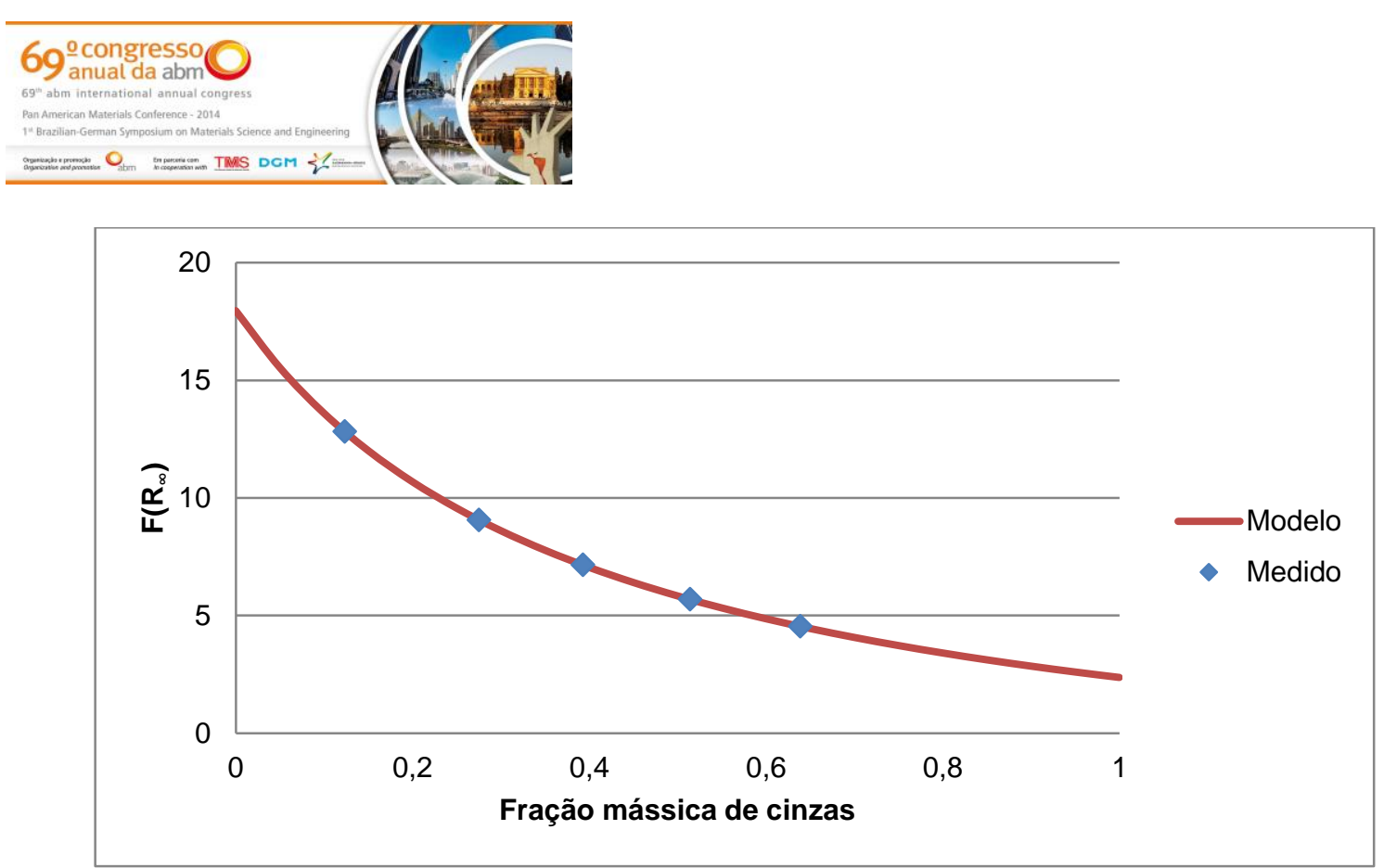

Figura 7: Modelo gerado a partir das amostras da camada I1 com $F\left(R_{\infty}\right)$ em $700 \mathrm{~nm}$.

O terceiro modelo gerado foi o modelo da camada de siltito que, igualmente à camada I1, contou com 5 pontos. Como resultado o modelo para as amostras da camada de siltito está exposto na figura 8.

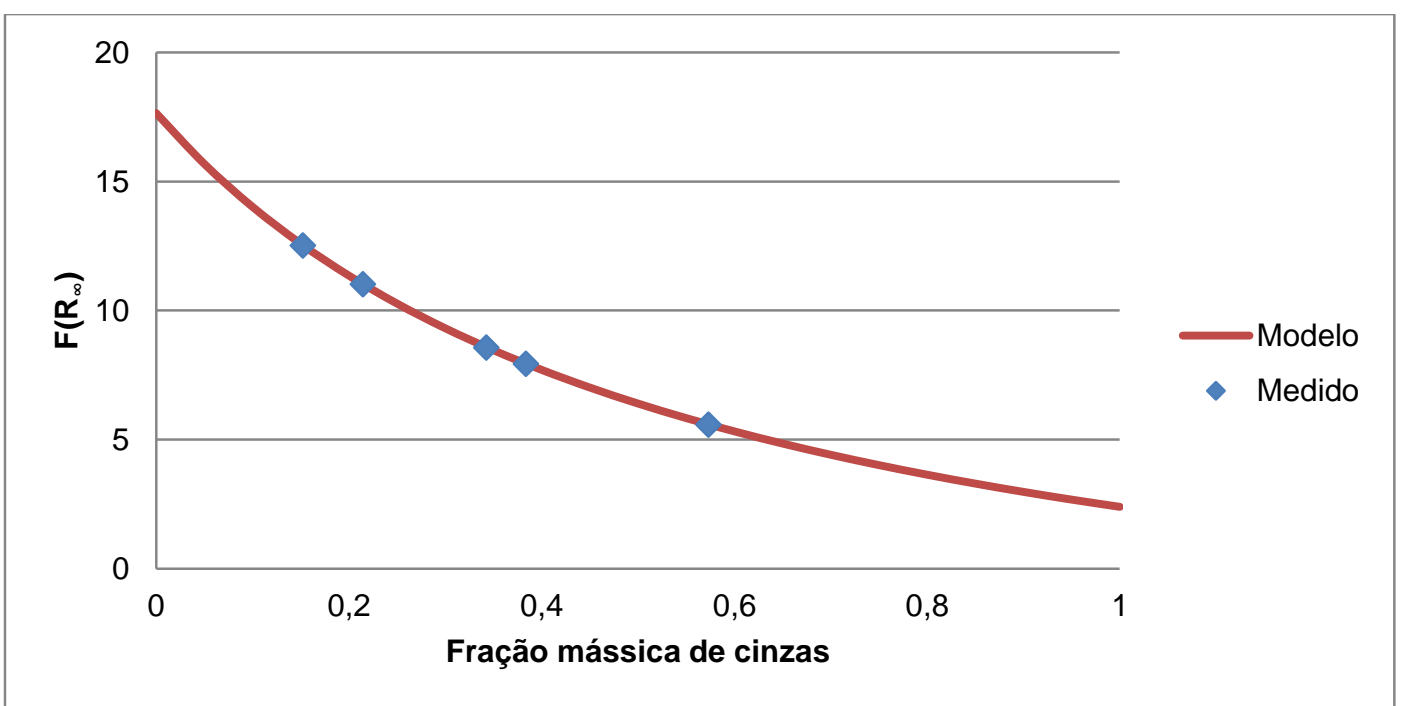

Figura 8: Modelo gerado a partir das amostras da camada de siltitos com $F\left(R_{\infty}\right)$ em $700 \mathrm{~nm}$.

A partir dos modelos gerados pode-se notar que foi possível adaptar com bastante exatidão o modelo exposto para as camadas individualmente separadas, conforme figuras 6, 7 e 8. Entretanto, o que pode explicar claramente esta ótima adaptação do modelo aos pontos amostrados pode ser a carência de pontos para geração do modelo. Em estudos posteriores o modelo a ser gerado terá como base uma maior gama de dados de forma a cobrir mais densamente e com maior amplitude a faixa de percentual de cinzas.

Após o cálculo dos três modelos gerados individualmente em cada uma das camadas foi gerado um quarto modelo que englobava as medidas realizadas em todas as camadas e como resultado se obteve o modelo da figura 9. Este modelo foi fruto do recálculo das constantes levando em conta todas as amostras.

\footnotetext{
* Contribuição técnica ao 69ำ Congresso Anual da ABM - Internacional e ao 14ํㅡㄹ ENEMET - Encontro Nacional de Estudantes de Engenharia Metalúrgica, de Materiais e de Minas, 21 a 25 de julho de 2014, São Paulo, SP, Brasil.
} 

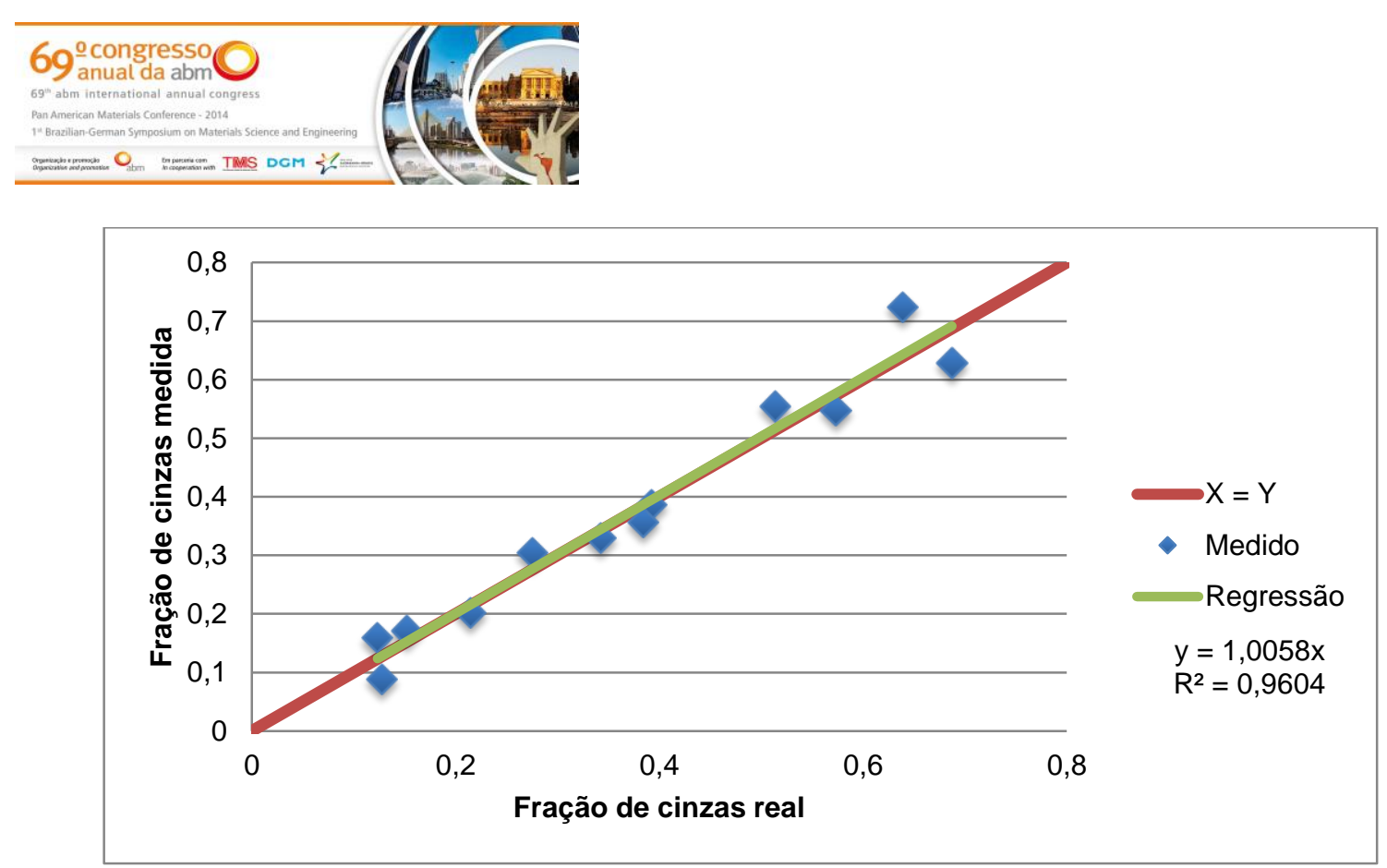

Figura 10: Gráfico de dispersão entre fração de cinzas medida e fração de cinzas real.

\section{CONCLUSÃo}

Os modelos propostos para as camadas individualizadas apresentaram resultados promissores, entretanto, há carência de dados que não transmite confiabilidade ao modelo. Já o modelo com todos as amostras agrupadas mostra um comportamento mais confiável uma vez que há uma razoável densidade de amostras por uma banda de valores de cinzas variando entre 15 e $70 \%$.

Apesar de mostrar uma maior gama de amostras o modelo com todas as amostras agrupadas também resulta nos maiores erros. Há claramente diferenças entre as constantes geradas nos 4 modelos diferentes o que leva a crer que existem diversos erros agregados às medidas.

Dentre os erros podem ser listados o erro fotométrico, erro devido ao baixo número de amostras e o erro devido à convenção utilizada de que o carvão é composto por apenas dois constituintes: cinzas e matéria orgânica. Entretanto, há uma forte correlação entre o percentual de cinzas medido pela via convencional e o medido a partir da reflectância pela teoria de K-M.

\section{Agradecimentos}

Nossos agradecimentos especiais à Copelmi Mineração Ldta. pelo apoio logístico, ao Conselho Nacional de Desenvolvimento Científico e Tecnológico - CNPq.

\section{REFERÊNCIAS}

1 Orel ZC. Application of Kubelka-Munk theory for the determination of the optical properties of solar absorbing paints. Process in Organic, 1997; 30: 59-66.

2 Christy AA. Quantitative analysis in diffuse reflectance spectroscopy: a modified Kubelka-Munk equation. Vibrational Espectroscopy, 1995; 9: 19-27.

3 Saunderson JL. Calculation of the color of pigmented plastics. Journal of the Optical Society of America, 1942; 32 .

4 Otsuka M. Comparative particle size determination of phenacetin bulk poder by using Kubelka-Munk theory and principal componente regression analysis based on nearinfrared spectroscopy. Poeder Technology,2004;141:244-250.

\footnotetext{
* Contribuição técnica ao $69^{\circ}$ Congresso Anual da ABM - Internacional e ao 14ํㅡㄹ ENEMET - Encontro Nacional de Estudantes de Engenharia Metalúrgica, de Materiais e de Minas, 21 a 25 de julho de 2014, São Paulo, SP, Brasil.
} 


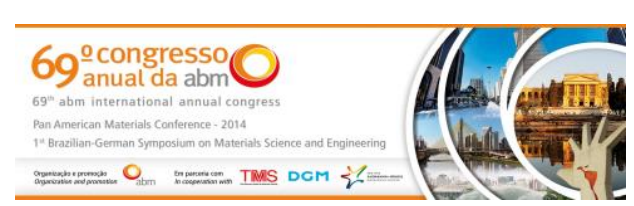

5 Osawa M. The measurement of meat pigments by fibre-optic reflectance spectrophotometry using the Kubelka-Munk equation. Meat Science, 1995; 40: 63-77.

6 Petter CO. (1994). Contribution à l'etude de la valorisation de kaolins pour l'industrie papetiere: mise au point d'une methodologie colorimetrique, application à la selectivité miniere. Paris: Tese de doutorado.

7 Peroni RL. (1998). Introdução de variável espectro-colorimétrica no modelamento e planejamento de jazidas de caolim e comparação da seletividade produzida por modelos de diferentes dimensões. Porto Alegre: Dissertação de mestrado.

8 Gonçalves I, Petter CO. Teoria de Kubelka-Munk aplicada na indústria de minerais industriais: predição do teor de contaminantes em caolim. Revista de Minas, 2007: 491496.

9 Abreu SF. (1973). Recursos Minerais do Brasil (Vol. II). Rio de Janeiro, RJ, Brasil: Edgar Blucher.

10 Gonçalve I. (2009). Determinação da concentração de contaminantes no caolin através da teoria de Kubelka-Munk. Porto Alegre: Dissertação de mestrado.

11 Souza V. Estimativa de qualidade de carvão por meio de perfilagem geofísica de gama natural eresistividade. Revista Escola de Minas, 2010; 63: 653-660.

12 Kortüm G. (1969). Reflectance spectroscopy. Principles, methods, applications. Springer-Verlag.

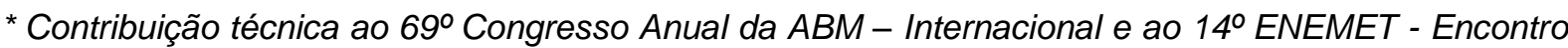
Nacional de Estudantes de Engenharia Metalúrgica, de Materiais e de Minas, 21 a 25 de julho de 2014, São Paulo, SP, Brasil. 